\title{
Stereotactic thalamotomy in the treatment of essential tremor of the upper extremity: reassessment including a blinded measure of outcome
}

\author{
A Zirh, S G Reich, P M Dougherty, F A Lenz
}

\begin{abstract}
The effectiveness of high frequency stimulation of the thalamic nucleus ventralis intermedius (Vim-HFS) for treatment of tremor has been studied by blinded assessment. The effectiveness of thalamotomy for essential tremor of the upper extremity by use of a blinded measure of outcome is now reported. Thalamotomy was performed in 21 patients (three operated on bilaterally) with medically intractable, essential tremor. Assessments of function, handwriting/drawing, and tremor amplitude were done before and at 3 and 12 months after surgery. The handwriting/drawing score was rated by a neurologist blinded to patient identity, laterality, and operative status. By comparison with baseline, both the total functional score and the total score from blinded assessment of handwriting/drawing improved significantly at 3 and 12 months after surgery. The two scores were significantly correlated, suggesting that the blinded assessment is a good predictor of a total disability from tremor. Complications after unilateral thalamotomy included transient dysarthria, permanent perioral numbness, and permanent mild disequilibrium in one patient each. Permanent mild dysarthria occurred in two of three patients operated bilaterally. Thus a blinded assessment of outcome establishes that unilateral thalamotomy is an effective, safe procedure for the treatment of essential tremor.

(F Neurol Neurosurg Psychiatry 1999;66:772-775)
\end{abstract}

Accepted 3 November 1998

Total functional, blinded handwriting/drawing and amplitude (action and posture) scores for essential tremor patients.

\begin{tabular}{lllll}
\hline $\begin{array}{l}\text { Average score } \\
\text { (mean (SD)) }\end{array}$ & Functional & $\begin{array}{l}\text { Blinded } \\
\text { handwwiting/ } \\
\text { drawing }\end{array}$ & $\begin{array}{l}\text { Postural tremor } \\
\text { amplitude }\end{array}$ & $\begin{array}{l}\text { Action tremor } \\
\text { amplitude }\end{array}$ \\
\hline $\begin{array}{l}\text { Pre-op } \\
\text { Post-op 3 months }\end{array}$ & $\begin{array}{l}4.4(1.8) \\
\text { Post-op 1 year }\end{array}$ & $6.3(0.7)$ & $3.0(0.3)$ & $3.5(0.2)$ \\
$\begin{array}{l}\text { Significance: } \\
\quad \begin{array}{l}\text { Pre-op v 3 months } \\
\text { Post-op v 1 year }\end{array}\end{array}$ & $\begin{array}{l}\mathrm{p}<0.001 \\
\mathrm{p}<0.001\end{array}$ & $\begin{array}{l}\mathrm{p}<0.8(0.4) \\
\mathrm{p}<0.001\end{array}$ & $\begin{array}{l}\mathrm{p}<0.7(0.4) \\
\mathrm{p}<0.05\end{array}$ & $\begin{array}{l}\mathrm{p}<0.6(0.4) \\
\mathrm{P}<0.05\end{array}$ \\
\hline
\end{tabular}

Keywords: microelectrode recording; thalamotomy; essential tremor; blinded assessment

Thalamotomy has been reported in small series to be an effective therapy for the treatment of medically intractable essential tremor of the upper extremity. ${ }^{1-5}$ Recently, high frequency stimulation of the thalamic nucleus ventralis intermedius (Vim-HFS) has emerged as a new surgical option for the treatment of essential tremor. $^{6-8}$ The first blinded study of the effectiveness of Vim-HFS has recently been published. ${ }^{7}$ To compare the effectiveness of thalamotomy with Vim-HFS, we now report the first study of thalamotomy for essential tremor using a blinded measure of outcome. An abstract of preliminary results has been published. ${ }^{9}$

\section{Methods}

Patients were selected for thalamotomy if they had medically intractable essential tremor. ${ }^{134}$ Patients were assessed before and at 3 and 12 months after surgery by a patient administered standard functional scale, standard handwriting/drawing scale, and a surgeon administered amplitude scale for tremor. ${ }^{10}$

The functional scale rated seven activities including speech, hygiene, eating, drinking, dressing, writing, and work. For each activity in the scale a score of 1 indicated normal function and 4 indicated the requirement of assistance to complete that activity. Thus the functional scale had a maximium of 28 (table, column 1) if the patient was totally disabled by tremor. The handwriting/drawing task was rated on a scale of 0 to 4 where 0 indicated normal, 2/4 indicated moderately abnormal writing and drawing that was obscured by multiple crosses of the line and $4 / 4$ indicated that the patient was unable to complete the task. The writing, drawing, and straight line tasks were scored separately for a maximum score of 12 (table, column 2). The amplitude of upper extremity tremor with posture and action were rated separately by the surgeon according to a four point direct scale: $0=$ no tremor, $1=$ amplitude $<0.5 \mathrm{~cm}, 2=0.5$ to $1 \mathrm{~cm}, 3=1$ to $2 \mathrm{~cm}$, $4=>2 \mathrm{~cm}$. Thus posture and action amplitude 
WRITE (with dominant hand only) "TODAY IS A NICE DAY IN BALTIMORE," SIGN YOUR NAME AND WRITE THE DATE JULY 4, 1776.
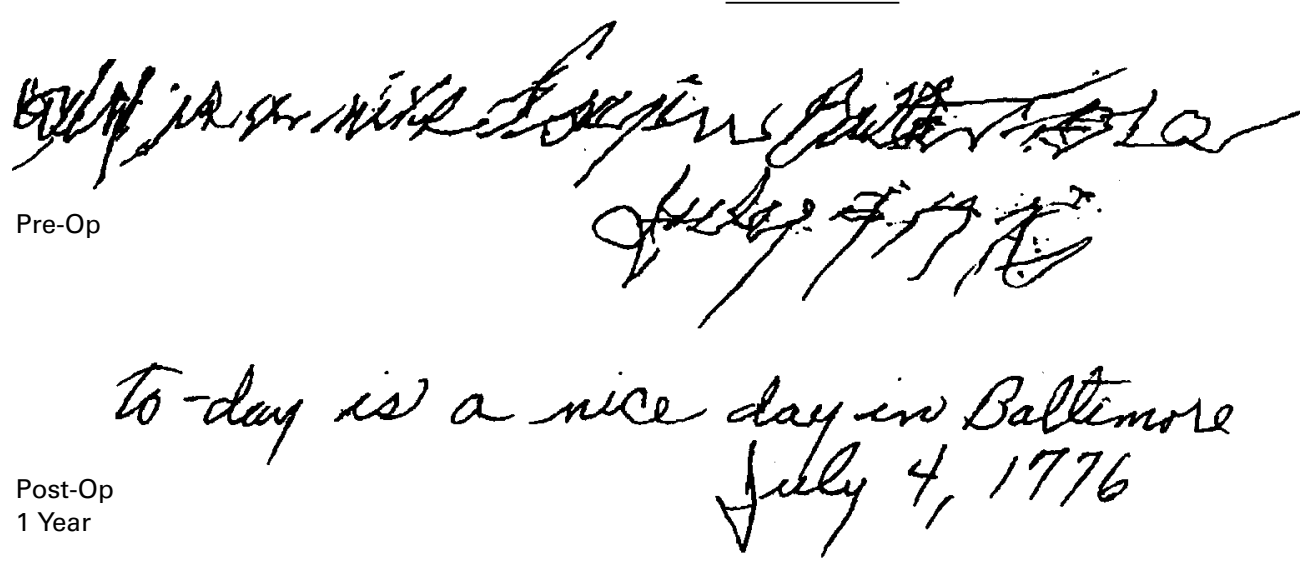

CONNECT THE DOTS ON EACH FIGURE WITH THE RIGHT HAND:

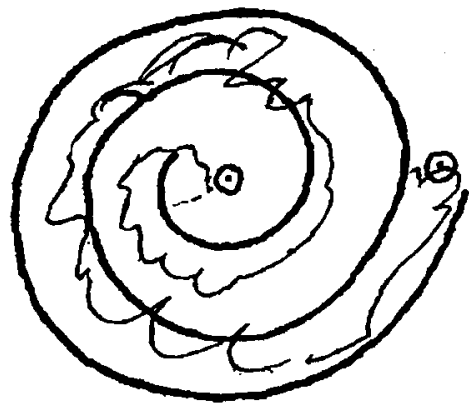

Pre-Op
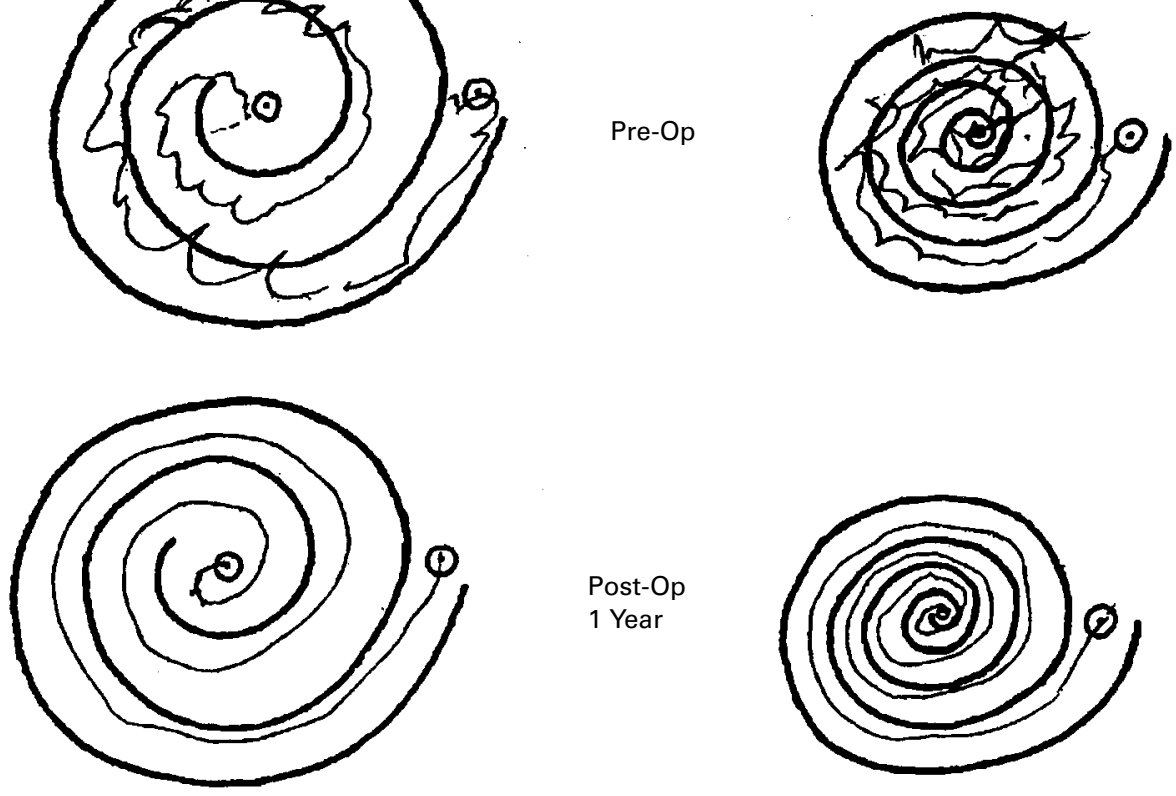

Example of the handwriting/drawing component of the standard rating scale of tremor. In each case the preoperative sample is shown above and the 1 year postoperative sample is shown below.

scores (table, columns 3 and 4) each had a maximum of 4 .

The handwriting/drawing component included writing (figure, upper panel), a spiral (lower panel), and straight line drawing task (not shown). In the second task a straight line is drawn between two parallel lines that are successively closer together in each of the three pairs of lines included in that task. The figure is included as an example of the scores obtained by use of the writing and spiral tasks. The drawing and spiral task are shown preoperatively and 1 year after a left thalamotomy. Both writing and the spiral drawing were scored at $3 / 4$ preoperatively. Postoperatively, writing was scored at $0 / 4$ and the spiral drawing task was scored at $1 / 4$.
The handwriting/drawing components of this scale were coded, ordered randomly, and then rated by a neurologist (SGR) who had not administered the rating scale but who was experienced in its interpretation. The neurologist was blinded to patient identity, laterality, and operative status. Statistical analyses of the functional, handwriting/drawing, and direct scales were carried out for the total score and the scores on each section (for example, spiral and straight line drawing sections) by a one way analysis of variance (ANOVA). Comparisons between intervals were adjusted for multiple comparisons (Dunn's multiple comparisons test).

As previously described, ${ }^{11}$ locations of thalamic nuclei were estimated radiologically and 
confirmed by microelectrode recording. The principal somatic sensory nucleus (ventralis caudalis: Vc) was identified as the region where most cells responded to deep or cutaneous sensory stimulation. ${ }^{12}$ The density of deep sensory cells was lower anterior to Vc, where cells with activity related to tremor were identified. ${ }^{13}{ }^{14}$ Sites where stimulation altered tremor were also identified anterior to the Vc. One or two radiofrequency lesions (table) were made anterior to the Vc among cells with activity related to tremor, deep sensory cells, and sites where microstimulation altered tremor.

Radiofrequency lesions were made with electrodes having a diameter of $1.1 \mathrm{~mm}$ and an exposed length of $3 \mathrm{~mm}$ (TM electrode - Radionics Inc, Burlington, MA, USA). Temperature was held constant at $60^{\circ} \mathrm{C}$ over a 1 minute interval and then increased by $5^{\circ} \mathrm{C}-10^{\circ} \mathrm{C}$ during subsequent 1 minute intervals to a final level of $80^{\circ} \mathrm{C}$. Neurological examination stressing pyramidal function, sensory function, cerebellar function, and speech was carried out before, during, and after each stage of the lesion procedure.

\section{Results}

We report the results of thalamotomy in all patients ( $n=21,13$ men, eight women) with essential tremor operated on by FAL between 1990 and 1993. The average age was 60.6 (SD 15.7) years and the average age of onset of tremor was 38.6 (SD 18.4) years. Eleven patients had familial essential tremor. All patients had failed treatment with propranolol and primidone both singly and in combination and most had failed treatment with clonazepam. Medications for the control of tremor were reduced after thalamotomy in 15 patients and were increased in none. The target side was the dominant hand in $14 / 21$ patients (right hand in 12/14). In three patients, a second thalamotomy was carried out more than a year later on the side opposite to the first procedure.

All complications were symptomatic and included: transient dysarthria of the cerebellar type after a left thalamotomy (one patient), permanent numbness about the contralateral angle of the mouth (one), and permanent, mild disequilibrium producing some uncertainty when the patient was dancing but not with other activities (one). Two of three patients operated bilaterally developed a mild, permanent dysarthria of the cerebellar type after the second procedure.

The table shows the mean (SD) of functional and blinded handwriting/drawing scores preoperatively and at 3 and 12 months postoperatively. Preoperative and postoperative functional scores were significantly different $(\mathrm{p}<0.001)$ from those at both 3 and 12 months postoperatively, indicating that the result of surgery was stable over this interval. On average, patients had moderate preoperative disability and zero to mild postoperative disability. By category (not shown) disability from working and writing (figure) were most significantly improved by surgery. The blinded handwriting/drawing scores indicate that dif- ferences between preoperative and both 3 and 12 months postoperative scores were significant $(p<0.05)$. On average, patients had moderately abnormal writing and multiple crosses of the line during drawing before surgery and zero to mild abnormality after surgery. Scores for postural and action tremor in the target side were significantly higher preoperatively than at both 3 and 12 months postoperatively. On the unoperated side, there was no significant difference between preoperative and postoperative tremor.

\section{Discussion}

Within patient improvement was assessed by comparing the mean of the preoperative and postoperative functional disability scores and the blinded scores. Ninety per cent of patients improved postoperatively. The mean of functional and blinded scores was significantly $(\mathrm{p}<0.05$, Mann-Whitney $U$ test) lower at 1 year after surgery in $72 \%(15 / 21)$ of patients. The total functional and blinded scores for each patient were highly correlated by linear regression $(r=0.72, \mathrm{p}<0.0001)$ which indicated that the blinded assessment of handwriting/ drawing was a good predictor of total disability from tremor.

Studies of small numbers of patients (6 to 13) having thalamotomy for essential tremor have been reported since the introduction of $\mathrm{CT}^{3415}$ These studies report no tremor or slight residual tremor in $66 \%$ to $100 \%$ of patients in the unblinded assessment of the surgeon or referring neurologist. Persistent complications occurred in $9 \%-28 \%$ with the most common being dysarthria, gait difficulties, weakness, and cognitive difficulties. There were no bleeds or wound problems but there was one death due to a pulmonary embolism. The magnitude of the effect reported in these studies is similar to that reported in the present blinded assessment

The present results are comparable with those of Vim-HFS as reported in an unblinded assessment of 20 patients with essential tremor. ${ }^{6}$ This assessment suggests complete disappearance of tremor or slight residual in $69 \%$ of patients at 3 months, and $59 \%$ at 6 months. A recent multicentre trial with blinded assessment using videotapes of 29 patients treated with Vim-HFS shows significant functional improvement associated with significant decreases in tremor by blinded assessment. ${ }^{7}$ By blinded assessment $90 \%$ of patients were improved by Vim-HFS in the latter study and by thalamotomy in the present study.

Complications of Vim-HFS included haematomas $(3 \%-5 \%)$, wound problems requiring reoperation $(3 \%)$, and one death from a pulmonary embolism. ${ }^{6}{ }^{7}$ Long term stimulation related side effects experienced with Vim-HFS included paraesthesias $(21 \%-9 \%)$, foot dystonia $(9 \%-4 \%)$, disequilibrium $(9 \%-4 \%)$, contralateral dystonia $(5 \%)$, dysarthria with unilateral implant $(20-0 \%)$, dysarthria with bilateral implants $(27.5 \%)$, and dysarthria with unilateral implant after thalamotomy (40\%). Although permanent complications of thalamotomy are higher than for Vim-HFS, the latter 
involves both implantation of a device and the long term maintenance of that device. ${ }^{16}$ Therefore, the present blinded assessment shows that thalamotomy is a safe and effective alternative to Vim-HFS for treatment of unilateral essential tremor of the upper extremity.

This work was supported by grants to FAL from the Eli Lilly Corporation and the NIH (P01 NS32386-Project 1).

1 Hirai T, Miyazaki M, Nakajima H, et al. The correlation between tremor characteristics and the predicted volume of effective lesions in stereotaxic nucleus ventralis intermedius thalamotomy. Brain 1983;106:1001-18.

2 Nagaseki Y, Shibazaki T, Hirai T, et al. Long-term follow-up results of selective VIM-thalamotomy. F Neurosurg 1986;65 296-302.

3 Goldman MS, Ahlskog JE, Kelly PJ. The symptomatic and medically intractable essential tremor. F Neurosurg 1992;76: 924-8.

4 Mohadjer M, Goerke H, Milios E, et al. Long term results of stereotaxy in the treatment of essential tremor. Stereotact Funct Neurosurg 1990;54+55:125-9.

5 Lakie M, Arblaster LA, Roberts RC, et al. Effect of stereotactic thalamic lesion on essential tremor. Lancet 1992;340:206-7.

6 Benabid AL, Pollak P, Gao D, et al. Chronic electrical Benabid AL, Pollak P, Gao D, et al. Chronic electrical
stimulation of the ventralis intermedius nucleus of the thastimulation of the ventralis intermedius nucleus of the tha-
lamus as treatment of movement disorders. $f$ Neurosurg 1996;84:203-14.
7 Koller W, Pahwa R, Busenbark K, et al. High frequency unilateral thalamic stimulation in the treatment of essential and parkinsonian tremor. Ann Neurol 1997;42:292-9.

8 Speelman JD, Bosch DA. Continuous electrical thalamus stimulation for the treatment of tremor resistant to pharmacotherapy. Ned Tijdschr Geneeskd 1995;139:926-30.

9 Lenz FA, Dougherty PM, Reich SG. The effectiveness of thalamotomy for treatment of tremor and dystonia thalamotomy for treatment of
[abstract]. Mov Disord 1996;11:18.

10 Fahn S, Tolosa E, Marin C. Clinical rating scale for tremor. In: Jankovic J, et al, eds. Parkinson's disease and movement disorders. Baltimore: Urban and Schwartzenberg, 1988: 225-34

11 Lenz FA, Normand SL, Kwan HC, et al. Statistical prediction of the optimal lesion site for thalamotomy in parkinsonian tremor. Mov Disord 1995;10:318-28.

12 Lenz FA, Dostrovsky JO, Tasker RR, et al. Single-unit analysis of the human ventral thalamic nuclear group: somatosensory responses. F Neurophysiol 1988;59:299-316.

13 Lenz FA, Kwan HC, Martin R, et al. Characteristics of somatotopic organization and spontaneous neuronal activity in the region of the thalamic principal sensory nucleus in patients with spinal cord transection. $f$ Neurophysiol in patients with spin $1994 ; 72: 1570-87$.

14 Lenz FA, Kwan H, Dostrovsky JO, et al. Single unit analysis of the human ventral thalamic nuclear group: activity correlated with movement. Brain 1990;113:1795-821.

15 Jankovic JJ, Cardoso F, Grossman RG, et al. Outcome after stereotactic thalamotomy for parkinsonian, essential and other types of tremor. Neurosurgery 1995;37:680-7.

16 Halpern MT, Palmer CS. Revised final report deep brain stimulation for the treatment of disabling tremor: clinical decision analysis model. Arlington, VA: Battelle, 1995:1-48. 